\title{
Preface
}

\section{Nutrient sensing and signalling in the gastrointestinal tract}

This issue of the British Journal of Nutrition publishes the proceedings of the symposium entitled 'Nutrient sensing and signalling in the gastrointestinal tract' organised by Shirazi-Beechey (University of Liverpool) and Bravo (Pancosma, Geneva). This symposium was held in Bratislava, Slovakia on 31 August 2012, as a part of the annual meeting of the European Association for Animal Production, and was sponsored by the Swiss Company Pancosma SA.

The major aim was to bring together key investigators in the field of gastrointestinal chemosensing, in order to provide a forum for stimulating discussion. The focus of the symposium was to learn about advances made in recent years on nutrient sensing and identify the potential for employing nutrient sensing receptors as nutritional targets for enhancing animal as well as human health.

The cells lining the surface of the intestinal epithelium are in direct contact with a luminal environment, the composition of which varies dramatically with diet. It has long been recognised that the gut is capable of sensing changes in luminal contents and responds by releasing chemical signals. In 1902, Bayliss and Starling ${ }^{(1)}$ noted that increasing the acidity in the small-intestinal lumen elicited pancreatic secretion, and that this was mediated, not via the nervous system, but by a humoral factor produced by the gut epithelium that they termed 'secretin'.

Indeed, we now know that the nerve endings that transmit signals evoked by changes in gut luminal contents do not reach the intestinal lumen and that the information about the chemical nature of the luminal contents is transmitted to neurons by enteroendocrine cells.

Enteroendocrine cells, dispersed among the cells lining the intestinal epithelium, represent approximately $1 \%$ of the entire gut epithelial population, but collectively they constitute the largest endocrine organ of the body ${ }^{(2)}$. They are flask-shaped, with the majority having long, slender apical processes that are in direct contact with the gut lumen, where they can 'sample' the luminal contents. Enteroendocrine cells are regarded as being pivotal to the chemosensing pathways of the intestinal tract. They respond to changes in gut contents by releasing hormones. Collectively, they produce over twenty different hormones, acting locally, centrally and in the periphery. There are at least ten discrete cell types that make up the enteroendocrine family, with each cell type having a distinct hormonal profile and localisation along the length of the gut ${ }^{(3)}$. Gut hormones play important roles in controlling vital physiological functions, such as nutrient digestion and absorption, food intake, insulin secretion and metabolism.

In recent years, significant advances have been made in the understanding of molecular recognition events involved in sensing the luminal contents of the gastrointestinal tract. There is convincing evidence that the sensing of various nutrients in the gastrointestinal tract is accomplished by a number of G-protein-coupled receptors, expressed on the apical membrane domain of enteroendocrine cells ${ }^{(4)}$. These include receptors for glucose, amino acids, protein hydrolysates, calcium and both long- and short-chain fatty acids. There are similarities between some of these receptors and taste receptors expressed in the lingual epithelium that mediate the detection of a number of tastants.

In this supplement of the British Journal of Nutrition, the proceeding four articles describe the mechanisms underlying nutrient sensing in the gastrointestinal tract and their role in initiating the pathways controlling various vital physiological processes.

The knowledge that gut epithelium can sense changes in the nutrient composition of the luminal contents through these cell-surface G-protein-coupled receptors assigns an important role for these receptors as accessible targets for manipulation. This has important potential for controlling many physiological processes, including nutrient digestion and absorption, food intake, insulin secretion and metabolism, leading to the maintenance of the health of the species.

Diet is also a major factor in driving the composition and metabolism of the intestinal microbiota. The amount, type and balance of nutrients has a great impact on the largeintestinal microbiota, which in turn majorly affect various aspects of host physiology ${ }^{(5)}$. For example, non-digestible food ingredients, referred to as prebiotics, have selective effects on influencing the intestinal microbiota. In this supplement, the potential of artificial sweeteners having a prebiotic-like effect and influencing the commensal gut microbiota is described by Daly et al. ${ }^{(6)}$ The identification of underlying mechanism(s) will assist in designing nutritional strategies for maintaining gut health.

\section{Acknowledgements}

We wish to thank Professor Jan Erik Lindberg and Dr Andrea Rosati for giving us the opportunity to organise this symposium within the annual meeting of the European Association for Animal Production, and Mr Daniel Kofel, Pancosma, for his continued support. The financial support 
of Pancosma SA for sponsoring this symposium is gratefully acknowledged. All authors wish to thank peer reviewers for their rigorous and critical assessment of manuscripts.

Soraya P. Shirazi-Beechey Institute of Integrative Biology University of Liverpool Liverpool, UK emailspsb@liverpool.ac.uk

David Bravo Pancosma SA, Geneva Switzerland

doi:10.1017/S000711451300233X

\section{References}

1. Bayliss WM \& Starling EH (1902) The mechanism of pancreatic secretion. J Physiol 28, 325-353.

2. Daly K, Darby AC, Hall N, et al. (2014) Dietary supplementation with lactose or artificial sweetener enhances swine gut Lactobacillus population abundance. Br J Nutr 111, S30-S35.

3. Sternini C, Anselmi L \& Rozengurt E (2008) Enteroendocrine cells: a site of 'taste' in gastrointestinal chemosensing. Curr Opin Endocrinol Diabetes Obes 15, 73-78.

4. Rehfeld JF (2004) A centenary of gastrointestinal endocrinology. Horm Metab Res 36, 735-741.

5. Wellendorph P, Johansen LD \& Bräuner-Osborne H (2010) The emerging role of promiscuous 7TM receptors as chemosensors for food intake. Vitam Horm 84, 151-184.

6. Scott KP, Gratz SW, Sheridan PO, et al. (2013) The influence of diet on gut microbiota. Pharmacol Res 69, 52-60. 